\title{
Aneurysm of the Right Atrial Appendage
}

\author{
Silvio Henrique Barberato, Márcia Ferreira Alves Barberato, Bianca Milanese Ávila, Sonia Perretto, \\ Liliam do Rocio Gavazzoni Blume, Miguel Chamma Neto \\ Curitiba, PR - Brazil
}

\begin{abstract}
Atrial aneurysms involving the free wall or atrial appendage are rare entities in cardiology practice and may be associated with atrial arrhythmias or embolic phenomena. We review the literature and report a case of aneurysm of the right atrial appendage in a young adult, whose diagnosis was established with echocardiography after an episode of paroxysmal atrial flutter.
\end{abstract}

Atrial aneurysms are extremely rare entities in cardiology practice. In the literature, we found 49 reported cases in the left atrium and only 7 in the right atrium ${ }^{1-2}$.

Left atrial aneurysms may be congenital and intrapericardial $^{3}$ or secondary to the partial absence of the pericardi$\mathrm{um}^{4}$ (due to herniation of the left atrial auricula). They most frequently manifest as recurring or incessant atrial arrhythmias $^{2}$, which may be refractory to medicamentous treatment and require surgical resection of the aneurysm. In addition, systemic embolization may occur as a severe complication ${ }^{5}$; therefore, long-term anticoagulation is indicated.

Right atrial aneurysms may be congenital and intrapericardial involving the free wall or they may result from trauma ${ }^{6}$. No report of aneurysm of the right atrial appendage exists in the literature. Patients may be asymptomatic ${ }^{7}$ or have atrial arrhythmias ${ }^{8,9}$ and repetitive pulmonary embolism ${ }^{10}$.

\section{Case report}

The patient is a 23-year-old male who sought emergency treatment complaining of rhythmic tachycardic palpitations of sudden onset. He reported a similar episode 4 years before, which was treated in the emergency department with intravenous medication. The physical examination was within the normal range, except for a heart rate of $150 \mathrm{bpm}$ and deviation of the ictus cordis to the left. The chest X-ray showed global enlargement of the cardiac area. The electrocardiogram diagnosed regular atrial flutter (type I), which

CEPEC - Centro Paranaense de Ecocardiografia, Hospital Universitário Cajuru da PUC-PR and Hospital Universitário Evangélico de Curitiba

Mailing address: Silvio Henrique Barberato - Rua Manoel Eufrásio, 403/4 - 80030440 - Curitiba, PR, Brazil - E-mail: silviohb@ cardiol.br

English version by Stela Maris C. e Gandour was successfully treated with chemical cardioversion with amiodarone. The following complementary examinations (thoracic echocardiogram, transesophageal echocardiogram and chest tomography) revealed a giant intrapericardial aneurysm of the right atrial appendage. On thoracic echocardiogram, the aneurysm measured $15 \times 8 \mathrm{~cm}$, caused compression of the middle and basal regions of the right ventricle and deviation of the cardiac structures to the left (fig. 1). Both ventricles were of normal size and function. The left atrium was normal. On Doppler, significant diastolic restriction to the filling flows of both ventricles was not found. During transesophageal echocardiography, intense spontaneous contrast (stasis) was detected inside the aneurysm, but with no thrombi (figs. 2, 3, and 4). No thrombi could be seen in the left atrium. Patent oval foramen was also diagnosed, with no hemodynamic repercussions.

The patient refused to undergo surgery, therefore, being kept on clinical medicamentous treatment with amiodarone and oral anticoagulant. Currently, the patient is asymptomatic and free from morbid events in the $9^{\text {th }}$ month of ambulatory follow-up.

\section{Discussion}

The case we report differs from those in the consulted literature in regard to anatomical features and clinical evolution. In regard to the location of the aneurysm, all those reported aneurysms were located in the trabecular portion of the right atrial free wall, anterior to the right ventricle. Our patient is the first reported with an aneurysm located specifically in the right atrial appendage, similar to the cases reported for the left atrial appendage (this location is more common for aneurysms of the left side). In regard to clinical evolution, even though the patient may be asymptomatic and the diagnosis established as a surgical or complementary examination finding, the most common occurrence was the arrhythmic manifestation. The resulting atrial arrhythmias evolve in an incessant or recurring way, and the potential risk of systemic or pulmonary embolic phenomena occurs. In the literature, we found reports of 2 asymptomatic patients. One was only diagnosed in the surgical suite ${ }^{7}$ during myocardial revascularization, when the aneurysm was resected and the patient evolved uneventfully; the other 


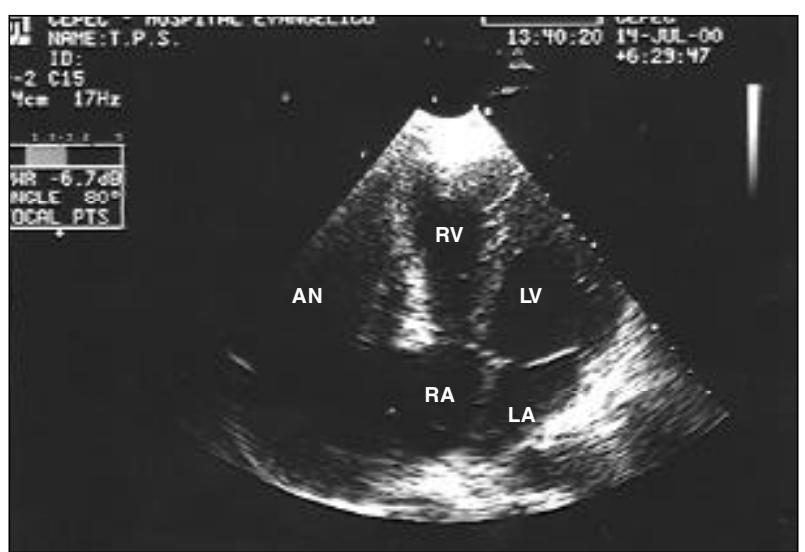

Fig. 1 - Transthoracic echocardiogram: aneurysm of the right atrial appendage producing compression of the right ventricle. RA- right atrium; AN- aneurysm; LV- left ventricle; LA- left atrium.

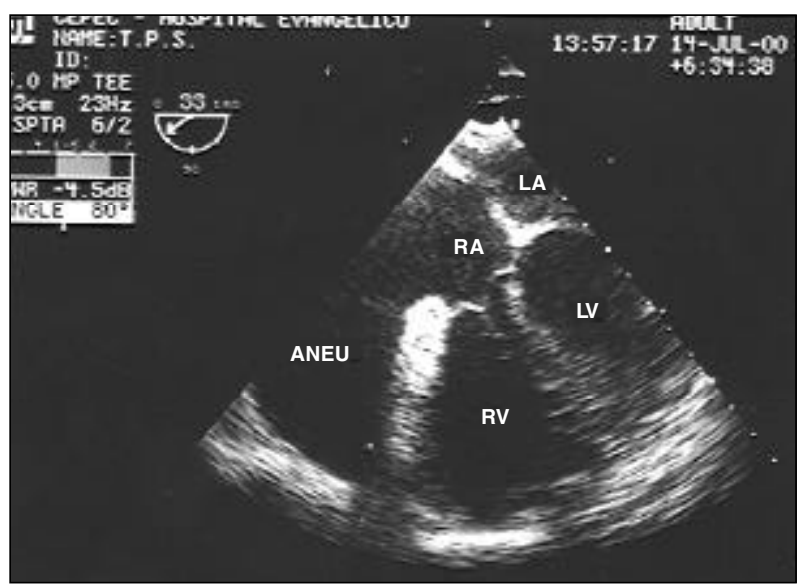

Fig. 2 - Transesophageal echocardiogram. RV-right ventricle; RA- right atrium ANEU- right atrial appendage aneurysm; LV- left ventricle; LA - left atrium.

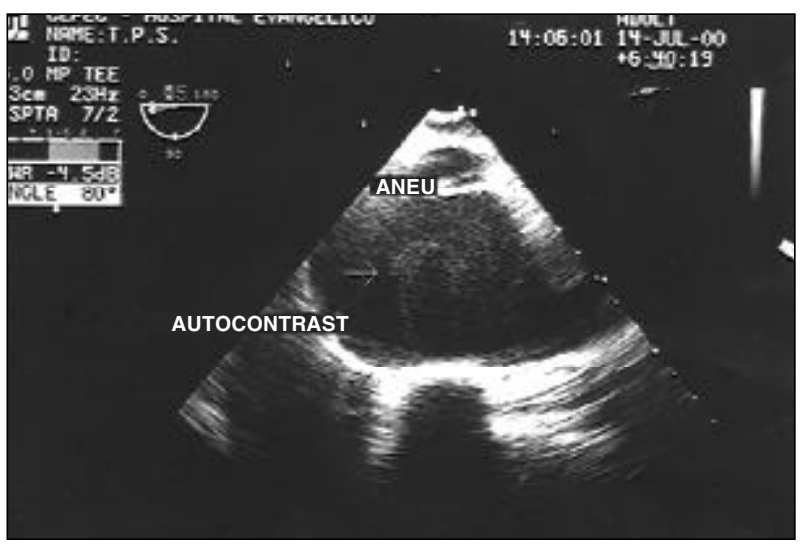

Fig. 3 - Transesophageal echocardiogram: intense auto-contrast (arrow tip) inside the aneurysm.

patient, whose diagnosis was an examination finding ${ }^{11}$, chose clinical management. One patient had a recurring pulmonary embolism ${ }^{10}$, was treated with oral anticoagulation, and remained asymptomatic for more than 4 years, when the case was published. One patient was diagnosed in the

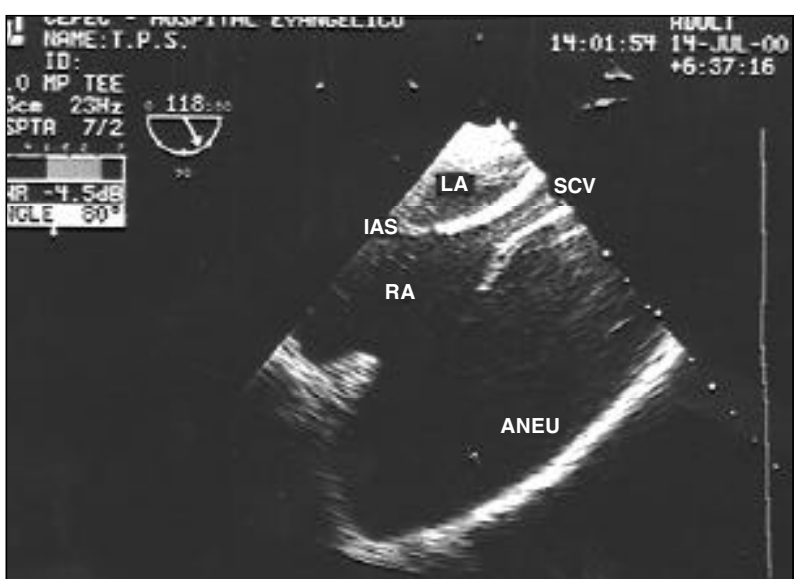

Fig. 4 - Transesophageal echocardiogram: longitudinal section showing relation of the aneurysm and atrial structures. LA- left atrium; IAS- interatrial septum; RA- right atrium; SCV- superior vena cava; ANEU- aneurysm of the right atrial appendage.

prenatal period, and gestation was interrupted ${ }^{12}$. The remaining 3 cases had incessant atrial arrhythmias ( 1 fibrillation, 1 flutter and 1 atrial tachycardia). Due to refractoriness to clinical treatment, the surgical treatment was indicated and resolved the arrhythmias found in 2 patients ${ }^{8-9}$. The unsuccessful case ${ }^{1}$, a patient with atrial fibrillation, also had 1 complication on the $4^{\text {th }}$ postoperative day, embolism to the anterior descending artery, which had not been recanalized by angioplasty, and new surgery was required. This event showed the need for surgical exploration of the left atrium in surgical resection of right atrial aneurysms, even when complementary examinations do not reveal thrombi. No death related to right atrial aneurysm has been reported in the literature. Our patient had only 2 episodes of arrhythmia during his 23 years of life; he evolved asymptomatically and not medicated until the $2{ }^{\text {nd }}$ episode. No manifestation of embolic phenomena could be detected through current or previous histories or on physical or complementary examinations. Because of the good clinical evolution, absence of incessant arrhythmia, and the patient's desire not to undergo surgery on that occasion, we chose medicamentous treatment with oral anticoagulants and antiarrhythmic agents, with follow-up with a complementary imaging examination every 6 months.

Despite the tendency found in the literature reports towards surgical indication, especially in symptomatic cases, the rarity of the affliction does not allow conclusions about the efficacy of surgery in curing arrhythmia and improving the prognosis of embolic events. We believe that, when facing such a rare affliction, individualization of the treatment according to clinical features (valuing the presence of incessant atrial arrhythmia and embolism) and complementary examinations (valuing the presence of thrombi, compression of adjacent structures, and associated diseases) is required.

In conclusion, aneurysm of the right atrium or atrial auricula is a rare malformation, which may evolve with high morbidity, and, therefore, should be remembered as a potential anatomic cause of atrial arrhythmias or embolic phenomena, or both. The diagnosis may be easily established through noninvasive complementary techniques, such as echocardiography. 


\section{References}

1. Suedkamp M, Horst M, Mehlhorn U, Hoppe U, Arnold G, Dalichau H. Surgical repair of right atrial aneurysm. Thorac Cardiov Surg 2000; 48: 35-7.

2. Gold JP, Afifi HY, Ko W, Horner N, Hahn R. Congenital giant aneurysm of the left atrial appendage: diagnosis and treatment. J Card Surg 1996: 11: 147-50.

3. Zimand S, Frand M, Hegesh J. Congenital giant left atrial aneurysm in infant. Eur Heart J 1997; 18: 1034-5.

4. Ruys F, Paulus W, Stevens C, Brutsaert D. Expansion of the left atrial appendage is a distinctive cross-sectional echocardiographic feature of congenital defect of pericardium. Eur heart J 1983; 4: 738 .

5. Gullestad L, Flogstad T, Nordstrand K, et al. Intrapericardial left atrial aneurysm diagnosed by transesophageal echocardiography and nuclear magnetic resonance imaging. Eur Heart J 1991; 12: 277-9.

6. Von der Emde J, Cesnjevar RA, Kretschmer S, Janssen GH, Wittekind C. Posttraumatic aneurysm of the right atrium. Ann Thorac Surg 1996; 62: 1507-9.
7. Zeebregts CJ, Hensens AG, Lacquet LK. Asymptomatic right atrial aneurysm: fortuitous finding and resection. Eur J Cardiothorac Sug 1997; 11: 591-3.

8. Scalia GM, Stafford WJ, Burstow DJ, Carruthers T, Tesar PJ. Successful treatment of incessant atrial flutter with excision of congenital giant right aneurysm diagnosed by transesophageal echocardigraphy. Am Heart J 1995; 129: 834-5.

9. Miyamura H, Nakagomi M, Eguchi S, Aizama Y. Successful surgical treatment of incessant automatic atrial tachycardia with atrial aneurysm. Ann Thorac Surg 1990; 50: 476-8.

10. Staubach P. Large right atrial aneurysm: rare cause of recurrent pulmonary embolism. Z Kardiol 1998; 87: 894-9.

11. Kozlj M, Angelski R, Pavcnik D, Zorman D. Idiopatic enlargement of the right atrium. Pediatr Cardiol 1998; 19: 420-1.

12. Gross B, Petrikovsky B, Challenger M. Prenatal diagnosis of an aneurysm of the right atrium. Prenat Diagn 1996; 16: 1034-5. 